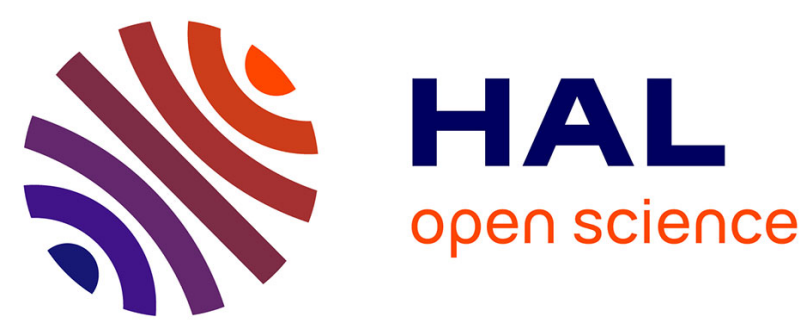

\title{
ÉTUDE EXPÉRIMENTALE DES GRANDEURS D'ENTRÉE D'UN RÉSONATEUR DE FORME SIMPLE COUPLÉ A UNE ANCHE SIMPLE
}

X. Meynial, Joel Gilbert, A. Hirschberg

\section{- To cite this version:}

X. Meynial, Joel Gilbert, A. Hirschberg. ÉTUDE EXPÉRIMENTALE DES GRANDEURS D'ENTRÉE D'UN RÉSONATEUR DE FORME SIMPLE COUPLÉ A UNE ANCHE SIMPLE. Journal de Physique IV Proceedings, 1992, 02 (C1), pp.C1-75-C1-78. 10.1051/.jp4:1992112 . jpa00251075

\section{HAL Id: jpa-00251075 https://hal.science/jpa-00251075}

Submitted on 1 Jan 1992

HAL is a multi-disciplinary open access archive for the deposit and dissemination of scientific research documents, whether they are published or not. The documents may come from teaching and research institutions in France or abroad, or from public or private research centers.
L'archive ouverte pluridisciplinaire HAL, est destinée au dépôt et à la diffusion de documents scientifiques de niveau recherche, publiés ou non, émanant des établissements d'enseignement et de recherche français ou étrangers, des laboratoires publics ou privés. 


\title{
ETUDE EXPERIMENTALE DES GRANDEURS D'ENTREE D'UN RESONATEUR DE FORME SIMPLE COUPLE A UNE ANCHE SIMPLE
}

\author{
X. MEYNIAL, J. GILBERT* ${ }^{*}$ et A. HIRSCHBERG* \\ Laboratoire d'Acoustique de l'Université du Maine, URA 1101 CNRS, Université du Maine, UFR \\ Sciences, Avenue Olivier Messiaen, BP. 535, F-72017 Le Mans cedex, France \\ *Laboratory for Fluid Dynamics and Heat Transfer, Department of Applied Physics, Eindhoven Uni- \\ versity of Technology, P.O. Box 513, NL-5600 MB Eindhoven, The Netherlands
}

\begin{abstract}
Résumé : Un dispositif permettant de simuler l'embouchure d'un clarinettiste ou d'un saxophoniste est présenté, ainsi que les capteurs donnant accès aux grandeurs d'entrées pertinentes pour les modèles d'oscillation. L'analyse des résultats met en évidence l'influence du débit "pompé" par l'anche. Des mesures en régime stationnaire sont comparées avec les modèles existants en vue de valider l'hypothèse quasi-stationnaire.
\end{abstract}

Abstract : A device simulating the embouchure of a clarinet or a saxophone player is presented together with the transducers deriving input signals relevant for the oscillations models. Analysis of the results shows the influence of the volume "pumped" by the reed. Measurements in stationnary mode are compared with the existing models in order to validate the quasi-stationnary hypothesis.

\section{Introduction}

Afin d'améliorer qualitativement et quantitativement la modélisation de l'oscillation des instruments à vent à anche simple (clarinette, saxophone) couramment utilisés dans la littérature, un montage a été réalisé donnant accès aux grandeurs d'entrée du système physique : la pression acoustique $p(t)$ dans le bec, le débit $Q(t)$ sous l'anche entrant dans le bec, le déplacement $y(t)$ de l'extrémité de l'anche.

\section{Description du dispositif expérimental}

Un bec de clarinette est enfermé dans une boite hermétiquement close, alimenté en air sous une pression statique $P$ ajustable. Afin d'être aussi proche que possible des conditions normales de jeu, "I'embouchure" de l'instrumentiste est représentée par un "étrier" sur lequel sont fixées des dents et une lèvre artificielle qui viennent appuyer sur l'anche. L'ensemble constitue ce, que l'on appelle "la bouche artificielle" (figure 1). Les résonateurs adaptés sur le bec de clarinette sont de forme simple, il s'agit soit de tubes cylindriques, soit d'un tube "monochromatique" (résonateur dont la géométrie est telle qu'il ne présente qu'une résonance majeure).

Un microphone piézo-céramique réalisé au laboratoire /1/, est affleurant à l'intérieur du bec. Supportant des niveaux élevés (jusqu'à $170 \mathrm{~dB})$, il permet de mesurer la pression acoustique dans le bec. La 
Un microphone piézo-céramique réalisé au laboratoire $/ 1 /$ est affleurant à l'intérieur du bec. Supportant des niveaux élevés (jusqu'à $170 \mathrm{~dB}$ ), il permet de mesurer la pression acoustique dans le bec. La position de l'anche au cours du temps est mesurée par un capteur capacitif, l'extrémité de l'anche métallisée jouant le rôle d'armature mobille. Ce capteur est calibré grâce à la présence d'un capteur optique. Ce dernier nous permet de visualiser le mouvement de l'extrémité de I'anche et l'ouverture anche-table du bec: un stroboscope piloté par un générateur basse fréquence ou par le signal de pression acoustique (signal éventuellement retardé d'une valeur ajustable), éclaire l'extrémité du résonateur; nous utilisons l'objectif macrozoom d'une caméra comme système optique grossissant d'un facteur 100 l'ouverture anche-table du bec. Si le débit moyen entrant dans 1'instrument est mesuré à l'aide d'un débitmètre à flotteur en série sur l'alimentation d'air, le débit instantané n'est pas mesurable directement. Nous mesurons une vitesse instantanée à l'entrée de l'instrument par une sonde à fil chaud.

Des mesures de $p(t), y(t)$ et $Q(t)$, cette dernière est la plus délicate. En effet, d'une part la sonde à fil chaud ne fonctionne pas en régime dynamique pour les faibles vitesses : typiquement pour $v<0.5 \mathrm{~m} / \mathrm{s}$, la convection libre devient prépondérante devant la convection forcée (la loi de King ne s'applique plus). D'autre part, la mesure du débit entrant suppose connu le profil de vitesse, de plus la vitesse mesurée est perturbée par une composante provenant, par l'extérieur, du mouvement de l'anche.

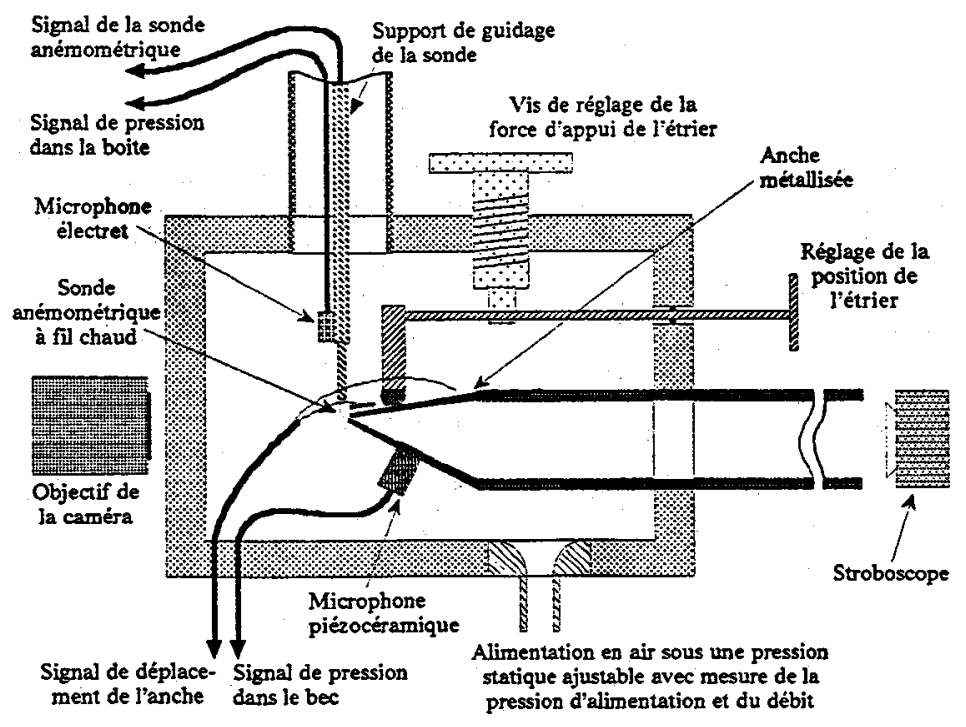

Figure 1 : "La bouche artificielle"-description du dispositif experimental.

\section{Résultats expérimentaux}

Nous présentons dans ce paragraphe quelques-uns des résultats de la quatrième partie de $/ 2 /$. 


\subsection{Evolution des signaux temporels en fonction de la pression d'alimentation $\mathbf{P}$.}

Après avois mis en évidence l'influence du débit pulsé par l'anche sur la mesure de la sonde à fil chaud en déplaçant cette dernière en différents points de l'entrée du bec, nous présentons figure 2, l'évolution des signaux d'entrée mesurés du seuil d'oscillation au seuil d'extinction.

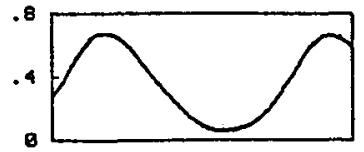

Cas (a)
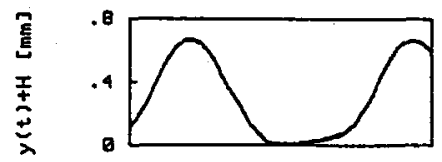

Cas (b)
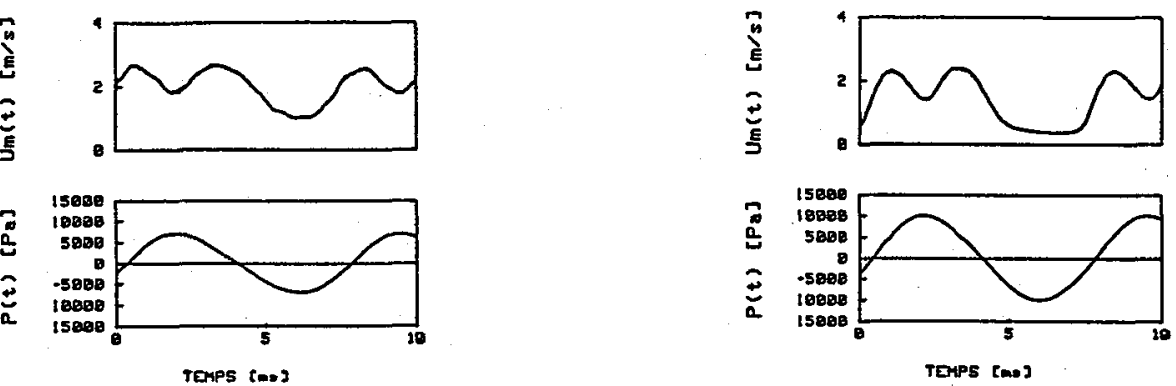

Figure 2 : Tracé des signaux mesurés de pression $p(t)$, de vitesse $u(t)$, de l'ouverture anche-table $y(t)+H$ (H est l'ouverture anche-table au repos). La pression d'alimentation est de $78 \mathrm{mB}$ (cas (a)), $104 \mathrm{mB}$ (cas (b)).

La pression acoustique $p(t)$ est toujours sinusoidale car nous utilisons le résonateur "monochromatique" présenté précédemment. Nous avons pu vérifier, grâce au capteur optique, que dans le cas (b) de la figure 2 l'anche se plaque contre la table du bec.

\subsection{Résultats expérimentaux en régime stationnaire}

Nous n'avons pas trouvé de manière évidente les deux types de comportement du jet entrant par le calcul anche-table du bec, exposés par Hirschberg et coll./3/. Dans le cas d'un bec 2D simplifié, les auteurs ont mis en évidence une séparation avec formation d'un "vena contracta" de surface approximativement moitié de la surface géométrique d'entrée et ensuite, soit recollement du jet sur les parois pour les faibles ouvertures $(\mathrm{H} / \mathrm{L}<0.25$, où $\mathrm{L}=1 \mathrm{~mm}$ est la longueur du canal d'entrée), soit formation de jet sans recollement sur les parois pour les grandes ouvertures $(\mathrm{H} / \mathrm{L}>0.5)$. Pour les grandes ouvertures, il y a un débit moitié par rapport au débit calculé selon la relation de Bernoulli car la surface transversale du jet est approximativement moitié de la surface géométrique d'entrée. Pour les faibles ouvertures, lorsque les frottements sont négligeables, le débit entrant approche le débit calculé selon Bernoulli, lorsque le frottement devient important, le débit entrant décroit pour tendre vers la valeur correspondant au débit calculé selon Poiseuille. 
Dans le cas d'un bec de clarinette, les résultats obtenus dépendent fortement de "1'embouchure" (position et pression de la lèvre sur I'anche). La dépendance de $Q / Q$ en fonction de $H$ est sans doute liée à la non prise en compte des fuites latérales (figure 3 ).

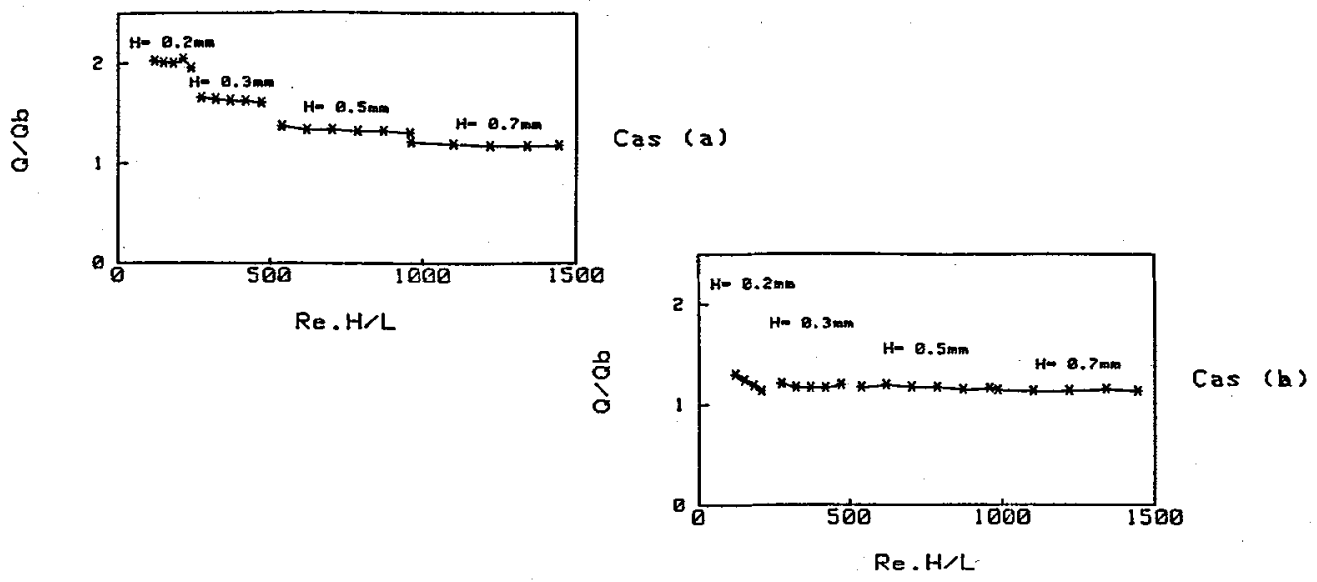

Figure 3 : Tracé du rapport sans dimension $Q / Q$ en fonction de la quantité sans dimension $R_{(}(H / L)$. Résultats expérimentaux pour quatre ouvertures anche-tăble $\mathrm{H}(0,7 \mathrm{~mm}, 0,5 \mathrm{~mm}, 0,3 \mathrm{~mm}$ et $0,2 \mathrm{~mm})$, pour deux positions de la lèvre sur l'anche. Cas (a), la lèvre est positionnée à $10 \mathrm{~mm}$ de l'entrée, Cas (b) la lèvre est positionnée à $5 \mathrm{~mm}$ de l'entrée.

\section{Conclusion}

L'écoulement $n$ 'est pas encore bien compris et l'expérience sur des géométries 3D proches de la réalité est essentielle. Néanmoins pour les grandes ouvertures une relation de type Bernoulli explique la relation débit-pression. Nous avons pu également justifier $/ 2 /$ l'hypothèse quasi-stationnaire en régime dynamique pour des nombres de Strouhal inférieurs à 0.01 , par superposition des résultats expérimentaux en régime stationnaire et en régime dynamique réalisés dans les mêmes conditions expérimentales.

\section{Références}

/1/ Meynial X., Systèmes micro-intervalles pour instruments à vent à trous latéraux ; Oscillation d'une anche simple couplée à un résonateur de forme simple. Thèse, Université du Maine, Le Mans (1987).

12/ Gilbert J., Etude des instruments de musique à anche simple : extension de la méthode d'équilibrage harmonique, rôle de l'inharmonicité des résonances, mesure des grandeurs d'entrée. Thèse, Université du Maine, Le Mans (1991).

/3/ Hirschberg A., Van-de-Laar R.W.A., Marrou-Maurrières J. -P. Wijnands A.P.J., Dane J.J., Kruijswijk S.G., Houtsma A.J.M., A quasistationary model of air flow in the reed channel of single reed woodwind insruments. Acustica 70 (1990), p. 146. 